\title{
Rapid Antibiotic Susceptibility Testing on Pathogenic Bacteria in Solid Growth Medium Using a Real-Time and Contactless Planar Microwave Resonator Sensor
}

\author{
Mandeep Chhajer Jain \\ University of British Columbia \\ Anupama Pillai \\ University of British Columbia \\ Rakesh Narang \\ University of British Columbia \\ Mohammad Zarifi ( $\square$ mohammad.zarifi@ubc.ca ) \\ University of British Columbia
}

\section{Research Article}

Keywords: Microwave resonator sensors, Bacteria detection, Antibiotic susceptibility testing

Posted Date: January 4th, 2021

DOl: https://doi.org/10.21203/rs.3.rs-135072/v1

License: (c) (i) This work is licensed under a Creative Commons Attribution 4.0 International License.

Read Full License

Version of Record: A version of this preprint was published at Scientific Reports on July 20th, 2021. See the published version at https://doi.org/10.1038/s41598-021-94139-y. 


\title{
Rapid Antibiotic Susceptibility Testing on Pathogenic Bacteria in Solid Growth Medium Using a Real-Time and Contactless Planar Microwave Resonator Sensor
}

Mandeep Chhajer Jain ${ }^{1}$, Anupama Pillai ${ }^{1}$, Rakesh Narang ${ }^{1}$, Mohammad Hossein Zarifi $1^{1 *}$

${ }^{I}$ Okanagan Microelectronics and Gigahertz Applications Laboratory, School of Engineering, University of British Columbia, Kelowna, BC VIV 1V7, Canada

Corresponding Authors:

Mohammad Hossein Zarifi, School of Engineering, The University of British Columbia, Kelowna, BC, V1V 1V7, Canada, Email: mohammad.zarifi@ubc.ca

\begin{abstract}
Infection diagnosis and antibiotic susceptibility testing (AST) are pertinent clinical microbiology practices that are in dire need of improvement, as current standards are not able to keep up with the mutations and resistance development of certain bacterial strains. This paper presents a novel way to conduct AST which hybridizes disk diffusion AST with microwave resonators for rapid, contactless, noninvasive and high-throughput testing. This work uses Escherichia coli (E. coli) cultured on solid agar and places bacteria samples on a microwave split-ring resonator along with antibiotic disks (erythromycin) of various doses to demonstrate the viability of this sensing method in a clinical microbiological setting. The microwave resonator, operating at a $1.76 \mathrm{GHz}$ resonant frequency, boasted a $5 \mathrm{~mm}^{2}$ sensitive sensing region. A one-port sensor was designed and optimized for detecting dielectric property variations of lossy dielectric materials accurately. This sensor was calibrated to detect uninhibited growth of the bacteria at $0.005 \mathrm{~dB} / \mathrm{hr}$, with a maximum change of $0.07 \mathrm{~dB}$ over the course of $15 \mathrm{hrs}$. The transient resonant amplitude change was subsequently dampened for each increasing dosage of antibiotic tested, with $45 \mu \mathrm{g}$ of erythromycin showing negligible change indicating complete inhibited growth. This AST sensor demonstrated decisive results of antibiotic susceptibility in under 6 hours and shows great promise to further automate the intricate workflow of AST in clinical settings, while providing rapid, sensitive, non-invasive and high-throughput detection capabilities.
\end{abstract}

Keywords: Microwave resonator sensors, Bacteria detection, Antibiotic susceptibility testing 


\section{Introduction}

Bacterial infections have become a public health crisis around the world in recent years. Due to over-prescription of antibiotics and non-compliance of their usage around the world, more families and strains of pathogens have begun to take a route towards further resistance against antibiotics ${ }^{1-4}$. Accordingly, the World Health Organization (WHO) has developed a list of 12 pathogen families which are highly resistant to antibiotics and pose a threat to public health on a global scale ${ }^{3}$. The new-found resistance of these families towards antibiotics, partnered with the WHO's bid towards further research into new antibiotics has also created a need for faster and more high-throughput antibiotic susceptibility testing (AST) practices ${ }^{4,5}$.

Current AST methods often suffer from the pitfalls of being extremely expensive, time consuming, labor intensive, prone to cross contamination, and have unstandardized practices ${ }^{5}$. Disk diffusion and broth dilution methods are currently the most widely utilized tests which use various antibiotics in different concentrations against bacterial colonies to test for antibiotic susceptibility or resistance ${ }^{4-8}$. These methods are extremely time-consuming and require upwards of 24 hours of incubation of the bacteria. Furthermore, they display poor performance when analyzing slow growing bacteria and both methods require expensive resources such as large volumes of reagents, and sizeable experimental equipment. Recently, matrixassisted laser desorption ionization-time of flight mass spectrometry (MALDI-TOF MS) has been deemed efficient in differentiating between various bacteria strains, however it is far too expensive to operate and maintain for most clinics globally ${ }^{9,10}$. Additionally, newly emerging genotypic methods have been used, which utilize polymerase chain reaction $(\mathrm{PCR})^{11,12}$, DNA microarrays ${ }^{13}$, or loop-mediated isothermal amplification $(\mathrm{LAMP})^{14}$, however their complex and user intensive protocols make them hard to implement in various clinics and situations ${ }^{4,11}$. Other emerging methods of AST which utilize fluorescence ${ }^{15}$, electrochemical sensors ${ }^{16}$, microfluidics ${ }^{17-21}$, microscopy ${ }^{22}$, and bioluminescence ${ }^{15}$ are currently improving scope of AST however, they are far too expensive and complex to develop, require laborious protocols to execute and need various expensive reagents and equipment making testing cumbersome ${ }^{4}$. This creates a 
crucial need for a more efficient and faster method of AST which is cheap enough to implement and does not require a plethora of resources to operate and maintain.

Microwave sensing is recently developing for clinical microbiology practices ${ }^{23-26}$. Microwave resonators have shown to be highly sensitive in sensing and monitoring bacteria ${ }^{25-28}$. Through translating variations of dielectric properties to quantifiable signals such has resonant amplitude and frequency, microwave sensing has shown to be adaptive towards the needs of biosensing and inexpensively implemented without the need to execute laborious protocols ${ }^{25,27,29,30}$. Planar microwave resonators are easily fabricated, can be reused for multiple assays, and have shown highly sensitive, real-time, and highthroughput results ${ }^{31-35}$. Microwave resonators have been coupled with microfluidic chips to simulate broth dilution tests and have already displayed high levels of sensitivity in monitoring bacteria in various conditions and monitoring bacterial growth ${ }^{25,26,36,37}$. Furthermore, differential microwave resonators have been implemented to reduce environmental noise in performing a more robust analysis on bioassays ${ }^{27,30}$. However, AST has only been proposed through microwave sensing and not yet demonstrated ${ }^{25}$. This lays on the premise in which antibiotics have proven to impact the growth of microorganisms by targeting the protein synthesis, DNA and RNA multiplication, and other metabolic activities to inhibit bacterial growth $^{38,39}$. The change of concentration of charged by products can indirectly aid in monitoring the metabolic activity by measuring conductivity and permittivity changes with resonant profiles overtime $^{25,26,28}$. Therefore, to tackle the need of faster and more efficient AST practices, microwave sensing can address all of the pitfalls suffered by the methods of AST previously discussed.

In this study, a simplified model of AST on Escherichia Coli (E. coli) using microwave split ringresonators is demonstrated through only using high concentrations of antibiotics, which the bacteria were proven to be susceptible to. Among the existing microstrip resonator variations, a frequency variation resonator consisting of a single split ring was employed to monitor and detect the impact of various concentrations of antibiotics on the growth of E. coli. Solid MH agar inoculated with E. coli is cultured on a microwave resonator ring gap and the transient resonant response of the resonator is dictated by the growth 
of the bacterial cells. Upon introducing various concentrations of the antibiotic erythromycin (E), bacteria growth is inhibited which deviates from the resonant response of this test as opposed to growing bacteria as seen in the decrease of variation in transient resonant amplitude change. This electrical signal is analyzed by a vector network analyzer (VNA) in a real-time, non-invasive and sensitive manner. Each sample was tested for 15 hours, however, decisive signals of growth inhibition was determined as early as 6 hours. This study not only calibrates a microwave resonator for AST on E. coli using erythromycin, but it demonstrates the efficacy of microwave sensors in clinical microbiological practices and has shown to vastly enhance the efficiency of AST.

\section{Results and Discussion}

\section{Electromagnetic Field Analysis of the Sensor}

A planar microstrip ring resonator structure was modelled in High Frequency Structure Simulator (HFSS) to study the operation of the resonator. The resonator was designed to operate at a frequency of $1.76 \mathrm{GHz}$ due to low effective loss of the solid MH agar media. The resonant structure consists of a halfwavelength split ring resonator and a feed line as shown in Figure 1(a). The microstrip resonator was designed on a low loss dielectric substrate (Rogers 5880) with permittivity of 2.2, loss tangent of 0.0009 , and thickness of $0.79 \mathrm{~mm}$ to minimize losses in the feed line or along the structure. The split ring resonator length was calculated using equation $(1)^{40}$ : 

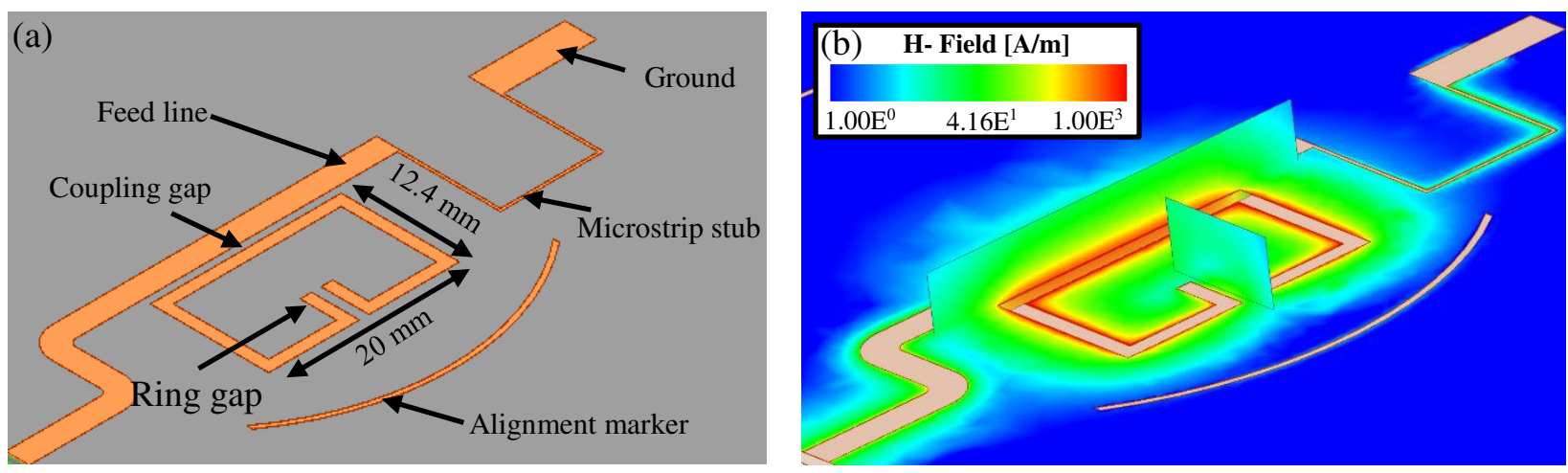
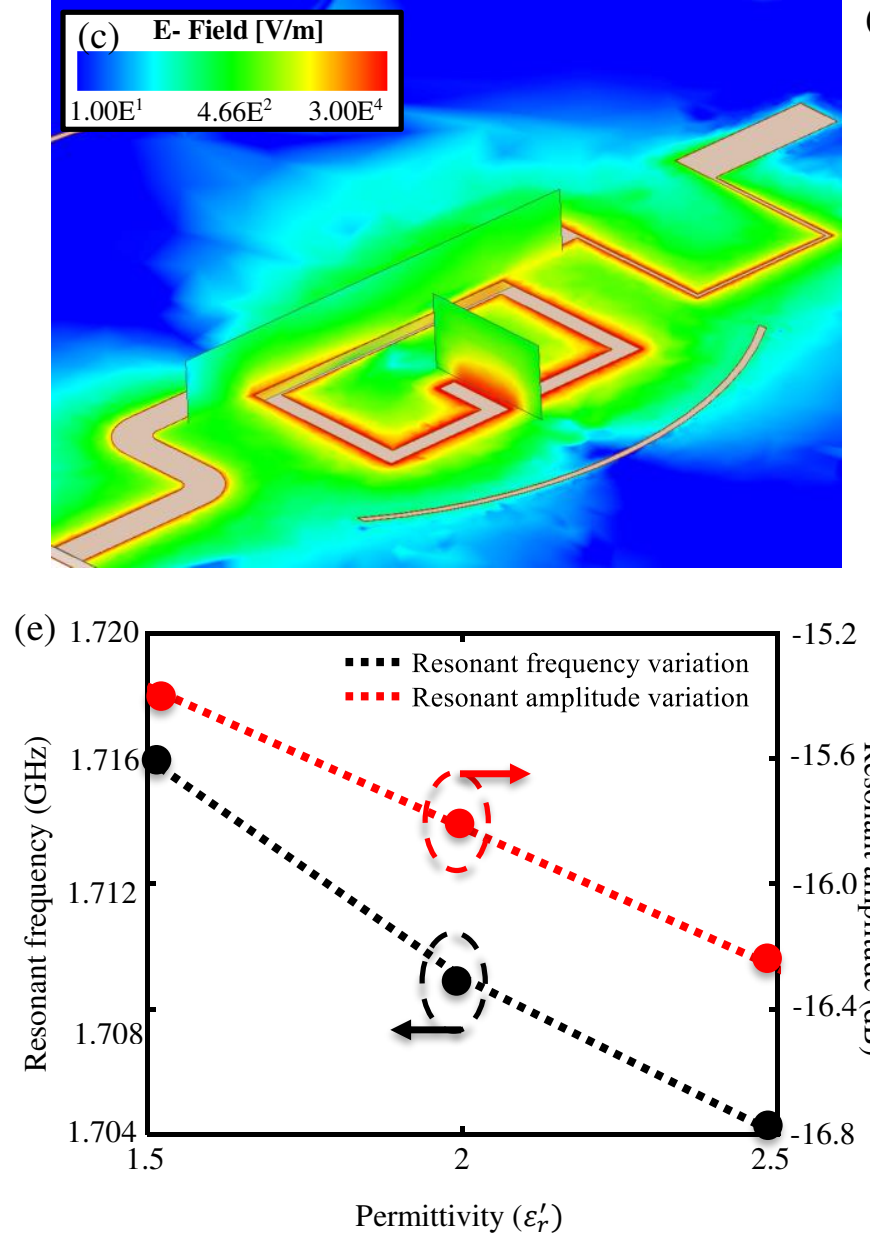
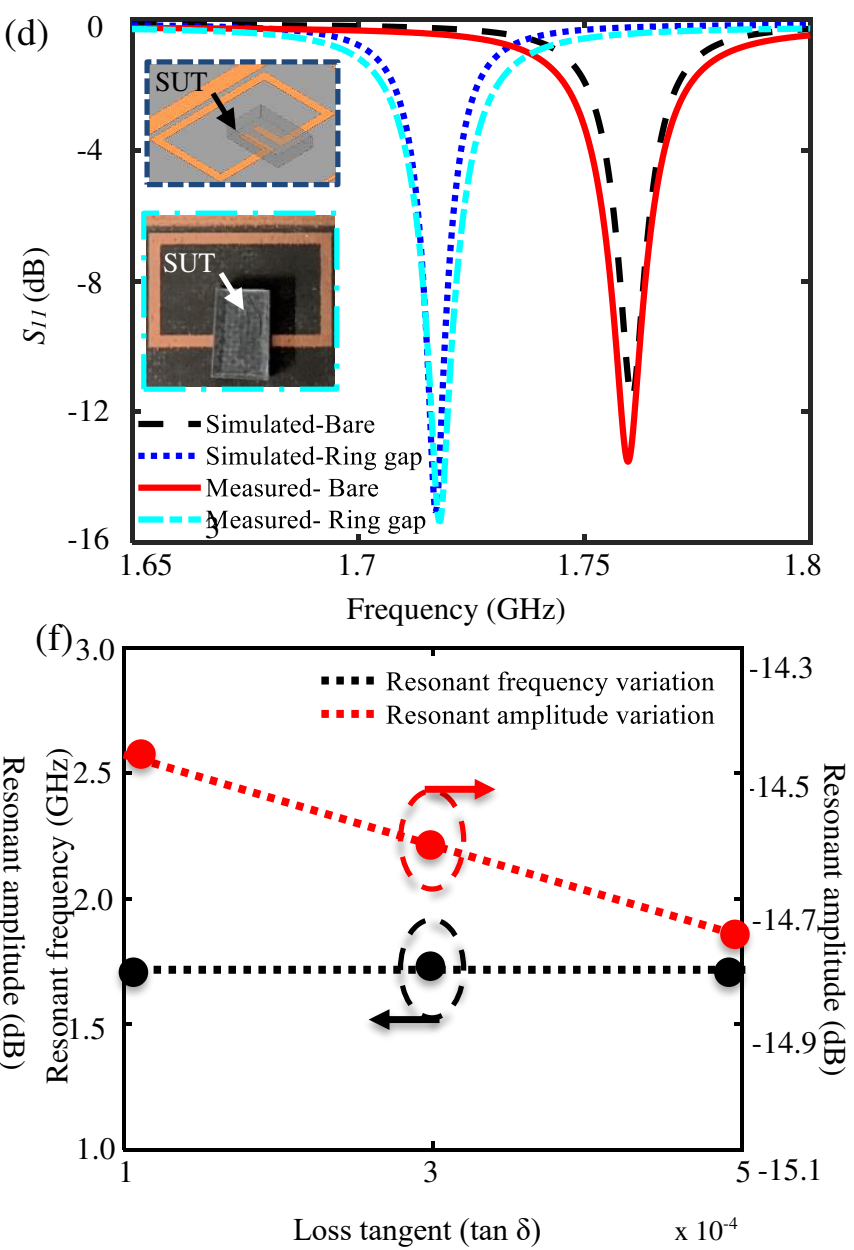

Figure 1. a) Modelled High frequency structure simulator (HFSS). b) Magnetic field distribution on the resonator. c) Electric field distribution on the resonator with the sensitive zone found in the ring gap. d) Simulated vs Measured reflection gain of the sensor in the absence and presence of a sample under test (SUT) on the sensitive region of the resonator. Impact of variation in e) permittivity, and f) loss tangent of the SUT on the resonant amplitude and resonant frequency of the resonator. 
$l=\frac{c}{2 f \sqrt{\varepsilon_{r}}}$

Where $l$ is the length of the split ring resonator $(\mathrm{m}), c$ is the speed of light $\left(3 \times 10^{8} \mathrm{~m} / \mathrm{s}\right), f$ is the resonant frequency $(1.76 \mathrm{GHz})$, and $\varepsilon_{r}$ is the relative permittivity of the substrate (2.2). Based on the equation (1), the length of the split ring resonator was calculated to be $57.5 \mathrm{~mm}$. However, resonant frequency is also governed by the capacitance of the resonant circuit ${ }^{40}$. Since, the ring gap of the resonator was modified to monitor an area of $5 \mathrm{~mm}^{2}$, the length of the resonator was modified to $73.8 \mathrm{~mm}$ to compensate the increase in the capacitive area and achieve the desired resonant frequency. The coupling gap width was selected as $0.8 \mathrm{~mm}$ to achieve maximum magnetic coupling between the feed line and the split ring as shown in Figure 1(b). The feedline was matched to the electromagnetic (EM) source impedance i.e. $50 \mathrm{Ohm}$ using LineCalc (by Advanced Design Systems 2020) to allow maximum power transfer from source to resonator structure. The dimensions of the feed line and the split ring resonator is shown in Figure 1(a).

The resonant structure was designed as a one-port device to reduce the complexity of the readout circuit. One port devices are widely used for accurately detecting and characterizing conductive dielectric materials ${ }^{41}$. As shown in Figure 1(a), one end of the feedline was connected to an EM source and the other end was terminated to ground using a high impedance quarter wavelength microstrip stub. The stub was added to discharge any weak capacitances formed between the feedline and the sample MH agar petri dish. The high impedance of the stub prevented loading of the matched feed line, while simultaneously acting as an open circuit to generate a microwave signal at the resonance frequency and short circuit at DC frequencies. An alignment marker was added in the design to strategically align the sample petri dish with the sensor. The simulated response of the bare sensor in the absence of test material is presented in Figure 1(d), shown in black, in which the resonant frequency and amplitude were found to be $1.76 \mathrm{GHz}$ and -11.58 $\mathrm{dB}$, respectively.

To determine the sensitive region of the resonator, a full 3D electromagnetic field simulation was performed in HFSS. The sensitive regions of the resonator are the regions displaying the maximum 
magnitude of electric field (E-field) shown in red within Figure 1(c). This figure presents the E-field distribution on the microstrip resonator sensor at the operating resonant frequency of $1.76 \mathrm{GHz}$. The electric field density was maximum around the ring gap as compared to the other regions of the sensor and was thus selected as the sensitive region of the resonator. Additionally, to validate the determined sensitive region, a system under test (SUT) was placed on the ring gap and the simulated response was analyzed as shown in Figure 1(d). For the SUT in this instance, a standard test material with a permittivity of 2.2, loss tangent of 0.0009 , and physical dimension of $6 \times 9 \times 1.57 \mathrm{~mm}$ was used. In effect, the overall effective dielectric properties at the sensitive region were modified resulting in a shift of the resonant profile of the resonator. The material was placed at a distance of $0.1 \mathrm{~mm}$ above the ring gap to further validate contactless sensing as a viable application for microwave-based sensing for AST. Based on the simulation results, a $-3.42 \mathrm{~dB}$ and $44 \mathrm{MHz}$ shift in the resonant amplitude and resonant frequency, respectively, was observed, indicating that the ring gap was the most sensitive to the presence of material and was chosen for further analysis.

In another study, an investigation to understand the impact of variation in the dielectric properties of the SUT on the response of the resonator was undertaken and the results are presented in Figure 1(e) and (f). The permittivity and loss tangent of the SUT were varied independently and the other physical properties of the material was held constant. Based on the simulation results, resonant amplitude and resonant frequency were found to be inversely proportional to variation in the permittivity of the SUT. However, variation in the loss tangent or conductivity only impacted the resonant amplitude of the sensor whereas, the resonant frequency remained unchanged. Since, bacterial growth significantly impacts the conductivity of the growth medium, resonant amplitude variations were selected as a dominant parameter to study the variation in the loss tangent or conductivity of the SUT.

Finally, the measured reflection gain of the fabricated resonator was measured and compared against the simulated results. In the absence of any test material, the resonant frequency and resonant amplitude of the fabricated sensor was found to be $1.76 \mathrm{GHz}$ and $-13.5 \mathrm{~dB}$, respectively, resulting in a $0.05 \%$ and $16.75 \%$ error between the simulated and measured values, for the resonant frequency and 
amplitude, respectively, which closely follows the simulation results shown in Figure 1(d). The deviation in the resonant amplitude between the simulated and measured values can be attributed to imperfections in the fabrication process which includes soldering of SMA, and the placement of via for ground. Furthermore, the sensitive region of the fabricated resonator was verified by placing a standard test sample (Rogers RT/duroid 5880) with a relative permittivity $\left(\varepsilon_{r}\right)$ of 2.2 , loss tangent $(\tan \delta)$ of 0.0009 , and dimensions of $6 \times 9 \times 1.79 \mathrm{~mm}$ on the ring gap of the resonator. A distinct shift in the resonant amplitude and resonant frequency by $-1.48 \mathrm{~dB}$ and $42 \mathrm{MHz}$, respectively, was observed when the material was placed on the ring gap, resulting in a $0.05 \%$ and $1.925 \%$ error between the experimental and theoretical values, which agrees with the simulation results shown in Figure 1(d). This confirmed the sensitivity of the selected region to the presence of test material and was therefore employed to monitor the impact of antibiotics on the growth of E. coli.

\section{Initial Antibiotic Susceptibility Disk Diffusion Calibration Discussion}

Prior to utilizing the microwave resonator to monitor the impact of antibiotics on the growth of microorganisms, a preliminary disk diffusion test was performed. In this test, the growth of $E$. coli was visually observed against various concentrations of erythromycin. Based on the captured images presented in Figure 2, different microbial responses were observed at various concentrations of erythromycin. Low concentrations of erythromycin (E-0 and E-7.5) did not inhibit microbial growth as there was no visible zones of inhibited growth. However, E-30 and E-45 resulted in the formation of zones of inhibited growth (171 $\mathrm{mm}$ and $280 \mathrm{~mm}$ respectively). This experiment verifies that E. coli $\mathrm{HB} 101$ is susceptible to E-30 and

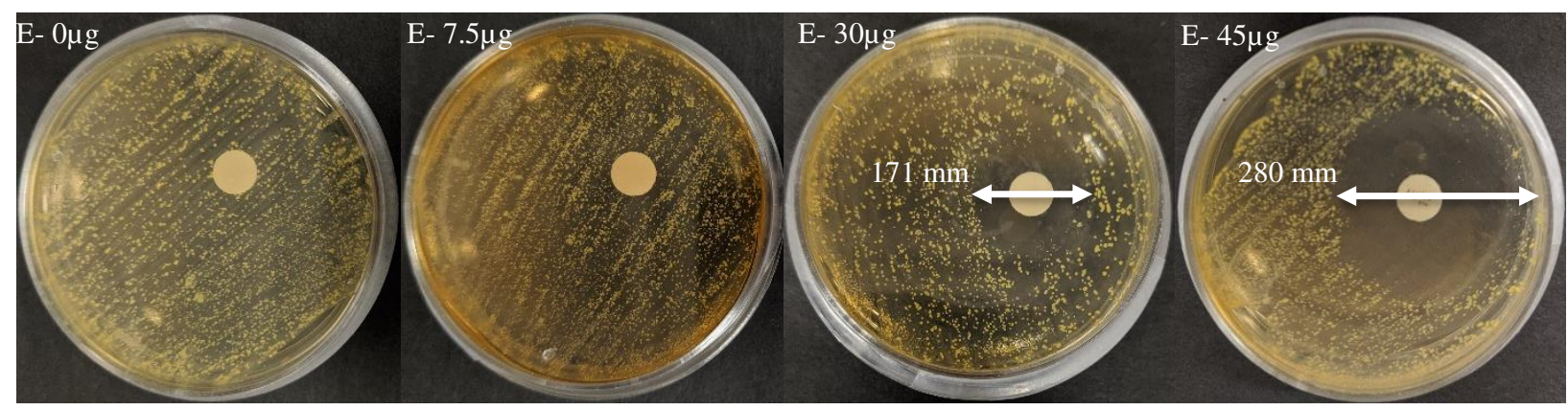

Figure 2. Captured images revealing the impact of $0,7.5,30$, and $45 \mu \mathrm{g}$ of erythromycin on the growth of E. coli. 
E-45, as used in this study. However, it should be noted that the bacteria is more susceptible to E-45 than E-30.

\section{Microwave and Image-based Detection of Bacterial Growth against Antibiotics}

A study to determine the impact of a hydrated blank paper disk (without antibiotics) and a $45 \mu \mathrm{g}$ erythromycin disk on the response of the resonator was performed to establish a baseline response to distinguish between detectable and undetectable regions of microbial growth (Figure 3(a)). It is evident from the measured results that the presence of a blank disk on a solid agar plate (with no bacteria) had negligible impact on the response of the resonator as opposed to the $\Delta$ Amplitude $(\mathrm{dB})$ measured for an inoculated solid agar plate with a blank disk $(\mathrm{E}-0 \mu \mathrm{g})$. The impact of an E-45 disk placed on a solid agar

plate (with no bacteria) on the response of the resonator is also presented in Figure 3(a). The measured resonant amplitude variation was insignificant in comparison to the measured resonant amplitude variation for a solid agar plate inoculated with bacteria and accompanied with a blank disk (E-0 $\mu \mathrm{g})$. Hence, the maximum variation in the resonant amplitude, i.e., $0.015 \mathrm{~dB}$, was considered as a threshold to determine the regions of undetectable and detectable bacterial growth.

Figure 3(b) presents the variation in the resonant amplitude corresponding to bacterial growth at tested antibiotic concentrations $(0,7.5,30$, and $45 \mu \mathrm{g})$. The figure shows a clear distinction between the 

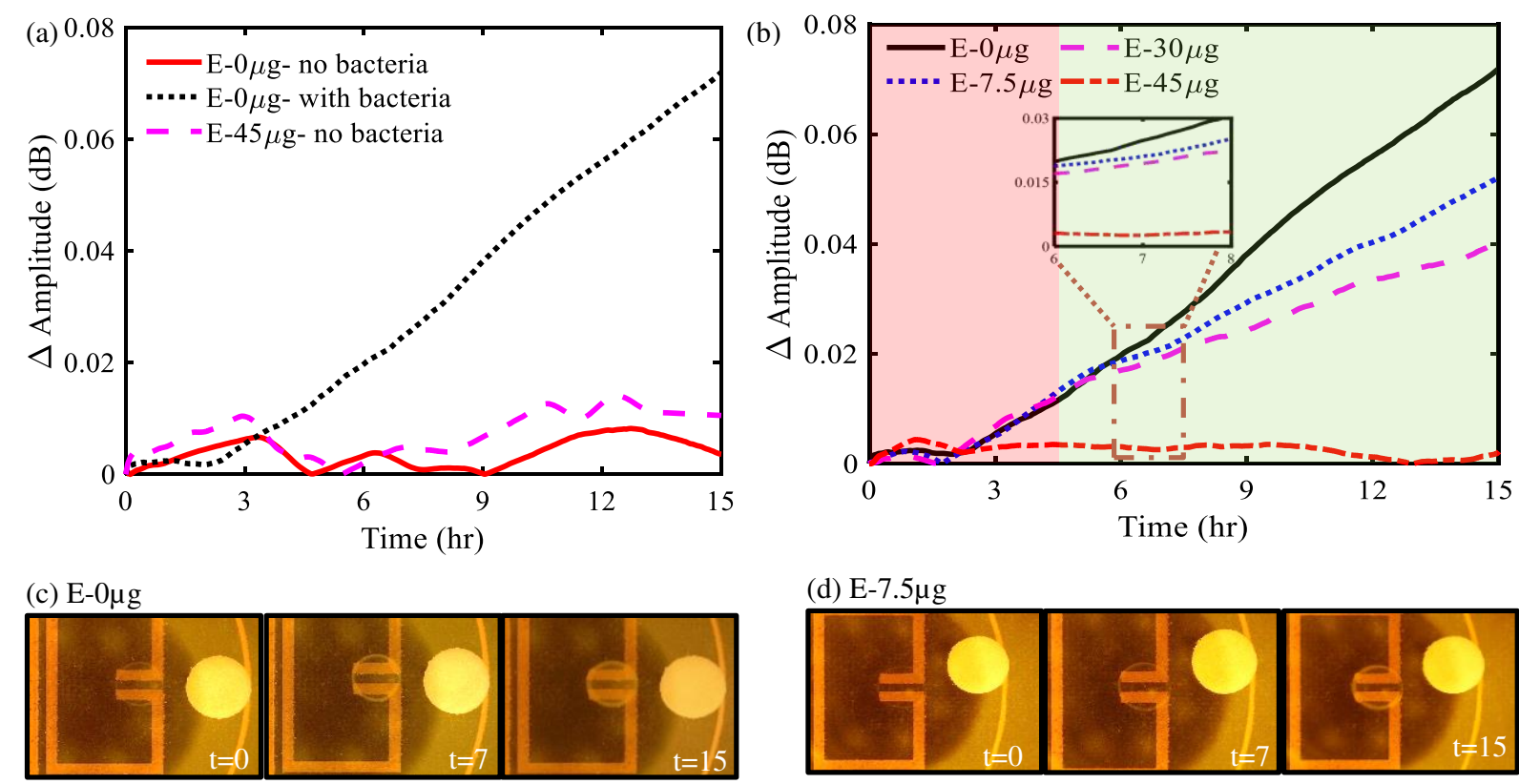

(d) $E-7.5 \mu \mathrm{g}$

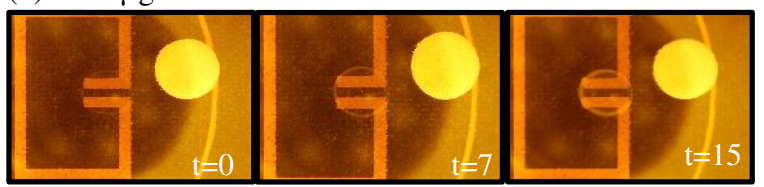

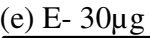

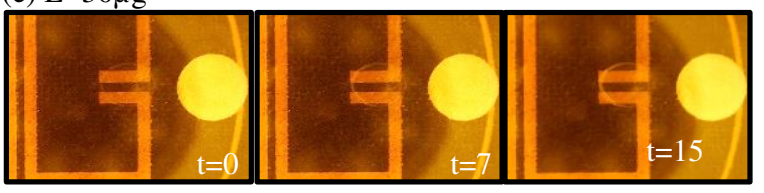

(f) E- $45 \mu \mathrm{g}$

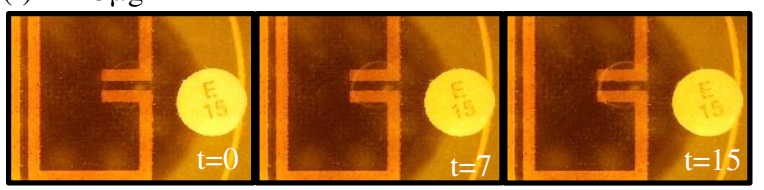

Figure 3: a) Impact of blank paper disk (without antibiotics) and a $45 \mu \mathrm{g}$ erythromycin disk on the response of the resonator. b) $\Delta$ resonant amplitude (dB) vs time (hr) depicting the detectable (green) and undetectable (red) regions of bacterial growth, (c)-(f) microscope images, of $0,7.5,30$, and $45 \mu \mathrm{g}$ of erythromycin.

measured E. coli growth at various concentrations of erythromycin. During bacterial growth, bacteria consume the nutrients readily available in their vicinity while synthesizing proteins and several charged byproducts ${ }^{42-47}$. The charged byproducts expelled into the surrounding media leads to conductivity changes in the growth medium, which is reflected in the resonant amplitude variation of the microstrip resonator sensor.

In the absence of erythromycin, a maximum resonant amplitude variation of $0.07 \mathrm{~dB}$ was observed, for the control plate $(\mathrm{E}-0 \mu \mathrm{g})$, due to unrestricted bacterial growth around the sensitive region of the sensor (Figure 3(c)). However, in the presence of erythromycin, the protein synthesis during bacterial metabolism was hindered, thereby impacting bacterial growth. As evident from Figure 3(b), the rate of change of resonant amplitude, which can be associated with the growth rate of bacteria decreased as the concentration 
of erythromycin increased, which is in positive correlation with the microscopic images captured at constant time intervals (Figure 3(c)-(f)). The slope of the measured resonant amplitude variation in the detectable bacterial growth region was calculated and found to be $0.005,0.003,0.002 \mathrm{~dB} / \mathrm{hr}$ with a coefficient of determination $\left(\mathrm{R}^{2}\right)$ of $0.999,0.998$, and 0.997 for $0,7.5$, and $30 \mu \mathrm{g}$ of erythromycin, respectively. Furthermore, an insignificant change in the $\Delta$ amplitude of $0.005 \mathrm{~dB}$ at $45 \mu \mathrm{g}$ erythromycin concentration indicated a complete inhibition of bacteria growth and was supported by microscopic images captured at constant time intervals (Figure 3(f)).

Bacterial susceptibility to antibiotics has shown to be concentration-dependent ${ }^{48,49}$. Low doses of erythromycin decrease protein synthesis in bacteria, thereby reducing the growth rate rather than inhibiting bacterial growth completely. This is in accordance with the bacteriostatic nature of erythromycin. However, higher concentrations of erythromycin completely inhibited bacteria growth leading to the formation of zones of inhibition as shown in Figure 2 in the previous experiement ${ }^{48-50}$. It is evident from the figure that a high concentration of erythromycin leads to a large zone of inhibition, and vice versa. Correlating these results with Figure 3, this explains not only the greater zone of inhibition for E-45 compared to E-30 and E-7.5, but also the difference of rates of change in the differential resonant amplitude over the course of the 15 hours measured by the microwave resonator. A noticeable difference can be seen in Figure 3(b), when comparing the differential responses of E-30 and E-7.5. Although subtle, E-30 showed a more dampened response of resonant amplitude change over time and had a lesser overall change than E-7.5, which is attributed to the reduced growth or metabolic activity of the bacteria. Therefore, in can be concluded that as the concentration of the erythromycin increased, the diameter of the zone of inhibition increased, overlapping the sensitive region of the microwave sensor. Consequently, the bacteria growth in the sensitive region decreased, which is in positive correlation with the measured microwave sensor's response and is supported by microscope images captured at constant time intervals (Figure 3(c) -(f)). Thus, it can be concluded that placing the antibiotic disks closer to the resonator will also impact the time at which decisive AST can be conclusive. However, the distance between the antibiotic disk and the resonator gap can be 
optimized as low concentrations marginally inhibiting growth can lead to inaccuracies for susceptibility in different concentrations.

A noteworthy outcome of this work was the sensor's ability to successfully distinguish the impact of different concentrations of erythromycin on the growth of E. coli before any visible cues. The microwave sensor was able to clearly distinguish the impact of erythromycin on E. coli growth within the first 6 hours of the experiment, which is far lower than the technical capacity of conventional microbiological disk diffusion studies. Early detection of the response of the microbial growth to antibiotics can be beneficial in developing treatment strategies against infections. Furthermore, the contactless, high-throughput, noninvasive, portable, inexpensive, and reusable nature of the planar microwave resonator makes it a promising tool in the field of microbiology.

In summary, a planar microwave resonator sensor was implemented to monitor the impact of antibiotics on the growth of microorganisms. The proposed sensing platform offers early detection of microbial growth by monitoring the conductivity variations of the growth medium due to microbial growth under the influence of antibiotics. The positive correlation between the measured microwave response of E. coli growth against the tested erythromycin concentrations along, with the microscopic images and the preliminary analysis of zones of inhibition, successfully demonstrated the efficacy of the proposed system. This work has shown promise for further automation of AST and increasing efficiencies within clinical microbiology labs for faster and more robust results for the proper care of bacterial infections.

\section{Methods and Materials}

\section{Reagents and Materials}

HB101 E.coli was used as the test microorganism which was cultured in Muller Hinton agar (C6421) (MH Agar) as the growth media was purchased from Hardy Diagnostics CRITERIONTM. BD BBL $^{\text {тM }} 15 \mu \mathrm{g}$ Erythromycin paper disk, BD BBL ${ }^{\text {TM }} 6 \mathrm{~mm}$ Blank paper disk, and MilliporeSigma ${ }^{\mathrm{TM}}$ Erythromycin (powdered form) was procured from Fisher Scientific, Canada. Ammonium persulfate as an 
etchant to fabricate microstrip sensor was purchased from MG Chemicals (Surrey, Canada). Dielectric laminates were provided by Rogers Corp. USA. Rohde and Schwarz ZNB20 VNA was used for the sensor measurement.

\section{Bacterial Samples Preparation}

Mueller Hinton Agar (MH agar) was used to prepare growth media used for the experiment. $1.91 \mathrm{mg}$ of $\mathrm{MH}$ agar was thoroughly mixed with $50 \mathrm{ml}$ of deionized water and sterilized for 15 minutes at $121{ }^{\circ} \mathrm{C}$ using an autoclave. The media was cooled down to $50{ }^{\circ} \mathrm{C}$ and $3 \mathrm{ml}$ of the sterilized media was poured into a Petri dish to achieve an agar thickness of $1 \mathrm{~mm}$. The plates were cooled down to room temperature and $3 \mu \mathrm{L}$ of E.coli with $\mathrm{OD}_{600}=1.5$ was inoculated at the predesignated position.

The antibiotic disks were prepared by soaking a blank paper disk with $30 \mu \mathrm{L}$ of the desired erythromycin concentration. The disk was transferred to the inoculated plate and placed at a constant distance of $10 \mathrm{~mm}$ from the inoculated $E$. coli. The Petri dish was sealed using a parafilm to avoid changes in the Petri dish due to external ambient factors.

\section{Fabrication of Microwave Resonator}

A microstrip planar ring resonator sensor was fabricated on a Rogers RO5880 laminate from Rogers Corporation Pvt. Ltd. with dielectric thickness of $0.79 \mathrm{~mm}$, copper cladding thickness of $35 \mu \mathrm{m}$, permittivity of 2.2 , and loss tangent of 0.0009 . The resonator pattern was etched following previously used protocols $^{37}$. Briefly, the split ring design was designed and simulated in ANSYS HFSS to optimize parameters and operating resonant profiles. Subsequently, the layout of the resonator was patterned using a laminator by incorporating several heating and pressing cycles. The patterned laminate was etched using ammonium persulfate. One end of the feedline was connected to a SMA connector and the other end was grounded. The modelled resonator structure and the simulation and fabrication results are discussed in detail in the above sections. 


\section{Initial Antibiotic Susceptibility Disk Diffusion Calibration}

To demonstrate the impact of $0,7.5,30$, and $45 \mu \mathrm{l}$ of erythromycin on the growth of E. coli, $\mathrm{MH}$ agar plates were inoculated using streak plate method. Blank paper disks were soaked in $30 \mu 1$ of RO water with 7.5, 30, and $45 \mu \mathrm{g}$ of erythromycin and tapped on the surface of the growth medium. The petri dish was sealed using parafilm to avoid precipitation and fungal contamination inside the petri dish. The petri dish was incubated at $22^{\circ} \mathrm{C}$ for 36 hours and the diameters of zone of inhibition was measured. The captured visual microbial growth at the end of this study are discussed in detail in the above sections.

\section{Experimental Setup of the Sensor and VNA}

The fabricated sensor arrangement shown in Figure 4(a) was enclosed in a thermally insulated and mechanically stabilized Styrofoam enclosure equipped with a LED light source, a digital microscope, and a temperature probe. The temperature probe was employed to monitor the variations in the ambient temperature. The ambient temperature was recorded to be $23.25 \pm 0.25^{\circ} \mathrm{C}$. The bacterial growth images were captured at fixed time intervals using a 10x Celestron digital microscope. The experimental setup is demonstrated in Figure 4(b).
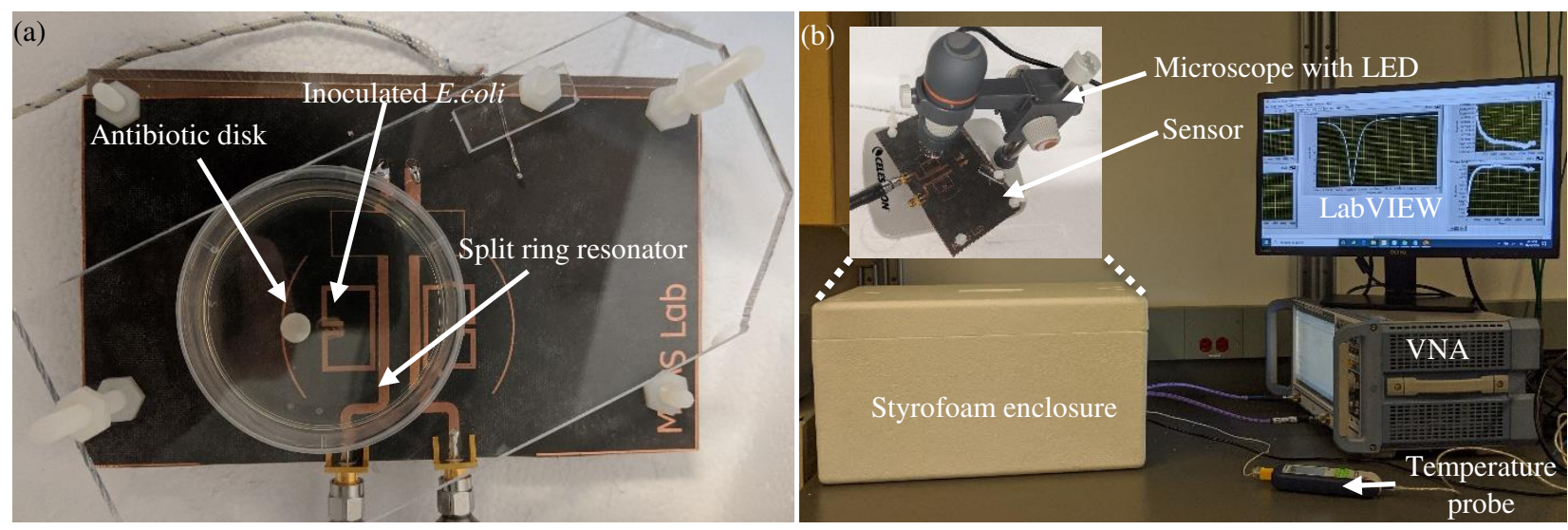

Figure 4. Fabricated sensor arrangement and the experimental setup for measurement of bacterial growth against antibiotics. a) The Fabricated planar microstrip resonator with sample petri dish placed strategically on the resonator.

b) Experimental setup consisting of a thermally insulated Styrofoam enclosure equipped with sensor, temperature probe, microscope with LED, and vector network analyzer (VNA). 
The inoculated plate was strategically positioned (Figure 4(a)) and firmly secured on the sensor such that the distance between the paper disk and the ring gap was $1 \mathrm{~cm}$. The sensor was connected to a ZNB20 Vector Network Analyzer (VNA) by Rohde and Schwarz. The VNA was calibrated every 15 hours to minimize the impact of drift in the measurement instrument on the response of the resonator. The VNA was operated between $1.55 \mathrm{GHz}$ to $1.8 \mathrm{GHz}$. The intermediate frequency (IF) bandwidth was set to $300 \mathrm{~Hz}$ to minimize the broadband noise, and the number of points was selected as 6401 to increase the sampling resolution. The output power of the VNA was set to $0 \mathrm{~dB}$ i.e. $1 \mathrm{~mW}$ which was sufficiently low to have no impact the growth of $E$. coli through adverse affects just as joule heating ${ }^{51,52}$. The VNA was triggered to measure the reflection coefficient $\left(S_{11} \mathrm{~dB}\right)$ every two minutes using an automated LabVIEW program made in house.

To test the ability of the sensor to monitor the susceptibility of E.coli to various concentrations of the antibiotic, erythromycin $(7.5,30$, and $45 \mu \mathrm{g})$, the reflection gain $\left(S_{11}(\mathrm{~dB})\right)$ of the sensor was measured and recorded using VNA and LabVIEW, respectively. The measured $S_{11}(\mathrm{~dB})$ was normalized by subtracting from the initially measured value due to day-to-day variations of ambient conditions. Furthermore, the data pertaining to the initial 90 minutes were excluded to account for stabilization of the measurement apparatus.

\section{Acknowledgements}

The authors would like to thank the Syilx Okanagan Nation for the use of their unceded territory, the land on which the research was conducted. We also acknowledge the partial financial support from the Natural Sciences and Engineering Research Council of Canada (NSERC), Discovery Grant Program, Canada Foundation for Innovation (CFI) program, and CMC Microsystems software and equipment. We also extend our gratitude to the Department of National Defence for funding support. 


\section{Authors Contribution}

M.C.J performed simulation modelling and designed the microwave resonator. M.C.J fabricated microwave resonator, A.P and M.C.J prepared bacteria and antibiotic samples and collected experimental data. M.H.Z. interpret the results, developed the idea and designed the experiments. M.C.J, R.N and M.H.Z wrote the manuscript.

\section{Competing Interest}

The authors declare that they have no competing interests.

\section{Bibliography}

1. Ventola, C. L. The antibiotic resistance crisis: causes and threats. $P$ T J. 40, 277-83 (2015).

2. Ayukekbong, J. A., Ntemgwa, M. \& Atabe, A. N. The threat of antimicrobial resistance in developing countries: Causes and control strategies. Antimicrobial Resistance and Infection Control vol. 6 (2017).

3. WHO publishes list of bacteria for which new antibiotics are urgently needed.

4. Khan, Z. A., Siddiqui, M. F. \& Park, S. Current and Emerging Methods of Antibiotic Susceptibility Testing. Diagnostics (Basel, Switzerland) 9, (2019).

5. Jorgensen, J. H. \& Ferraro, M. J. Antimicrobial susceptibility testing: A review of general principles and contemporary practices. Clinical Infectious Diseases vol. 49 1749-1755 (2009).

6. Sader, H. S. \& Pignatari, A. C. E test: a novel technique for antimicrobial susceptibility testing. São Paulo medical journal = Revista paulista de medicina vol. 112 635-638 (1994).

7. Bauer, A. W., Kirby, W. M., Sherris, J. C. \& Turck, M. Antibiotic susceptibility testing by a standardized single disk method. Am. J. Clin. Pathol. 45, 493-496 (1966).

8. Guardino, R. F. Early History of Microbiology and Microbiological Methods.

9. Hrabák, J., Chudác ková, E. \& Walková, R. Matrix-assisted laser desorption ionization-time of flight (MALDITOF) mass spectrometry for detection of antibiotic resistance mechanisms: From research to routine diagnosis. Clin. Microbiol. Rev. 26, 103-114 (2013).

10. Bernardo, K. et al. Identification and discrimination of Staphylococcus aureus strains using matrixassisted laser desorption/ionization-time of flight mass spectrometry. Proteomics 2, 747-53 (2002).

11. Cockerill, F. R. Genetic methods for assessing antimicrobial resistance. Antimicrobial Agents and Chemotherapy vol. 43 199-212 (1999).

12. Park, S., Zhang, Y., Lin, S., Wang, T. H. \& Yang, S. Advances in microfluidic PCR for point-ofcare infectious disease diagnostics. Biotechnology Advances vol. 29 830-839 (2011).

13. Frye, J. G. et al. Development of a DNA microarray to detect antimicrobial resistance genes identified in the national center for biotechnology information database. Microb. Drug Resist. 16, 9-19 (2010).

14. Fluit, A. C., Visser, M. R. \& Schmitz, F. J. Molecular detection of antimicrobial resistance. Clinical Microbiology Reviews vol. 14 836-871 (2001).

15. Webb, J. S. et al. Green Fluorescent Protein as a Novel Indicator of Antimicrobial Susceptibility in Aureobasidium pullulans. Appl. Environ. Microbiol. 67, 5614-5620 (2001).

16. Liu, T., Lu, Y., Gau, V., Liao, J. C. \& Wong, P. K. Rapid Antimicrobial Susceptibility Testing with Electrokinetics Enhanced Biosensors for Diagnosis of Acute Bacterial Infections. Ann. Biomed. 
Eng. 42, 2314 (2014).

17. Yasaki, H. et al. A real-time simultaneous measurement on a microfluidic device for individual bacteria discrimination. Sensors Actuators, B Chem. 260, 746-752 (2018).

18. Boedicker, J. Q., Li, L., Kline, T. R. \& Ismagilov, R. F. Detecting bacteria and determining their susceptibility to antibiotics by stochastic confinement in nanoliter droplets using plug-based microfluidics. Lab Chip 8, 1265 (2008).

19. He, J. et al. A novel microbead-based microfluidic device for rapid bacterial identification and antibiotic susceptibility testing. Eur. J. Clin. Microbiol. Infect. Dis. 33, 2223-2230 (2014).

20. Tang, Y., Zhen, L., Liu, J. \& Wu, J. Rapid Antibiotic Susceptibility Testing in a Microfluidic pH Sensor. Anal. Chem. 85, 2787-2794 (2013).

21. Chen, C. H. et al. Antimicrobial Susceptibility Testing Using High Surface-to-Volume Ratio Microchannels. Anal. Chem. 82, 1012-1019 (2010).

22. Baltekin, Ö., Boucharin, A., Andersson, D. I. \& Elf, J. Fast Antibiotic Susceptibility Testing ( FASTest ) based on single cell growth rate measurements. Proc. Natl. Acad. Sci. 114, 9170-9175 (2017).

23. Chen, Y.-F., Wu, H.-W., Hong, Y.-H. \& Lee, H.-Y. $40 \mathrm{GHz}$ RF biosensor based on microwave coplanar waveguide transmission line for cancer cells (HepG2) dielectric characterization. Biosens. Bioelectron. 61, 417-421 (2014).

24. Kim, N. Y., Dhakal, R., Adhikari, K. K., Kim, E. S. \& Wang, C. A reusable robust radio frequency biosensor using microwave resonator by integrated passive device technology for quantitative detection of glucose level. Biosens. Bioelectron. 67, 687-693 (2015).

25. Narang, R. et al. Sensitive, Real-time and Non-Intrusive Detection of Concentration and Growth of Pathogenic Bacteria using Microfluidic-Microwave Ring Resonator Biosensor. Sci. Rep. 8, 15807 (2018).

26. Mohammadi, S. et al. Real-time monitoring of Escherichia coli concentration with planar microwave resonator sensor. Microw. Opt. Technol. Lett. 61, 2534-2539 (2019).

27. Jain, M. C., Nadaraja, A. V., Vizcaino, B. M., Roberts, D. J. \& Zarifi, M. H. Differential Microwave Resonator Sensor Reveals Glucose-Dependent Growth Profile of E. coli on Solid Agar. IEEE Microw. Wirel. Components Lett. 1-4 (2020) doi:10.1109/LMWC.2020.2980756.

28. Oberoi, S., Daya, K. S. \& Tirumalai, P. S. Microwave sensor for detection of E. coli in water. in 2012 Sixth International Conference on Sensing Technology (ICST) 614-617 (IEEE, 2012). doi:10.1109/ICSensT.2012.6461753.

29. Mohammadi, S. et al. A Label-Free, Non-Intrusive, and Rapid Monitoring of Bacterial Growth on Solid Medium Using Microwave Biosensor. IEEE Trans. Biomed. Circuits Syst. 14, 2-11 (2020).

30. Mohammadi, S., Nadaraja, A. V., Roberts, D. J. \& Zarifi, M. H. Real-time and hazard-free water quality monitoring based on microwave planar resonator sensor. Sensors Actuators A Phys. 303, 111663 (2020).

31. Zarifi, M. H. \& Daneshmand, M. Monitoring Solid Particle Deposition in Lossy Medium Using Planar Resonator Sensor. IEEE Sens. J. 17, 7981-7989 (2017).

32. Zarifi, M. H. Sensitivity and Selectivity Enhancement in Coupling Ring Resonator Sensors Using Splitting Resonant Frequencies. in 2018 IEEE/MTT-S International Microwave Symposium - IMS 36-39 (IEEE, 2018). doi:10.1109/MWSYM.2018.8439564.

33. Rafi, M. A., Wiltshire, B. D. \& Zarifi, M. H. Wideband Tunable Modified Split Ring Resonator Structure Using Liquid Metal and 3-D Printing. IEEE Microw. Wirel. Components Lett. 30, 469472 (2020).

34. Kozak, R., Wiltshire, B. D., Khandoker, M. A. R., Golovin, K. \& Zarifi, M. H. Modified Microwave Sensor with a Patterned Ground Heater for Detection and Prevention of Ice Accumulation. ACS Appl. Mater. Interfaces 12, 55483-55492 (2020).

35. Muñoz-Enano, J., Vélez, P., Gil, M. \& Martín, F. Planar Microwave Resonant Sensors: A Review and Recent Developments. Appl. Sci. 10, 2615 (2020).

36. Accelerating bacterial growth detection and antimicrobial susceptibility assessment in integrated 
picoliter droplet platform. doi:https://doi.org/10.1016/j.bios.2017.06.006.

37. Zarifi, M. H., Sadabadi, H., Hejazi, S. H., Daneshmand, M. \& Sanati-Nezhad, A. Noncontact and Nonintrusive Microwave-Microfluidic Flow Sensor for Energy and Biomedical Engineering. Sci. Rep. 8, 139 (2018).

38. Kohanski, M. A., Dwyer, D. J. \& Collins, J. J. How antibiotics kill bacteria: from targets to networks. Nat. Rev. Microbiol. 8, 423-435 (2010).

39. Jurtshuk, P. Bacterial Metabolism. Medical Microbiology (1996).

40. Pozar, D. M. Microwave engineering; 3rd ed. (Wiley, 2005).

41. Ray J, K. Microwave Reflection Resonator Sensors. (1994).

42. Jurtshuk, P. Bacterial Metabolism. Medical Microbiology (1996).

43. Sanchis, A. et al. Dielectric characterization of bacterial cells using dielectrophoresis. Bioelectromagnetics 28, 393-401 (2007).

44. Bai, W., Zhao, K. S. \& Asami, K. Dielectric properties of E. coli cell as simulated by the three-shell spheroidal model. Biophys. Chem. 122, 136-142 (2006).

45. Schaechter, M. A brief history of bacterial growth physiology. Front. Microbiol. 6, (2015).

46. Allison, J. B., Anderson, J. A. \& Cole, W. H. The Method of Electrical Conductivity in Studies on Bacterial Metabolism. J. Bacteriol. 36, 571-86 (1938).

47. Negrete, A., Ng, W. I. \& Shiloach, J. Glucose uptake regulation in E. coli by the small RNA SgrS: Comparative analysis of E. coli K-12 (JM109 and MG1655) and E. coli B (BL21). Microb. Cell Fact. (2010) doi:10.1186/1475-2859-9-75.

48. Bernier, S. P. \& Surette, M. G. Concentration-dependent activity of antibiotics in natural environments. Front. Microbiol. 4, 20 (2013).

49. Udekwu, K. I., Parrish, N., Ankomah, P., Baquero, F. \& Levin, B. R. Functional relationship between bacterial cell density and the efficacy of antibiotics. J. Antimicrob. Chemother. 63, 745757 (2009).

50. Kapoor, G., Saigal, S. \& Elongavan, A. Action and resistance mechanisms of antibiotics: A guide for clinicians. J. Anaesthesiol. Clin. Pharmacol. 33, 300-305.

51. Banik, S., Bandyopadhyay, S. \& Ganguly, S. Bioeffects of microwave-a brief review. Bioresour. Technol. 87, 155-159 (2003).

52. Jankovic, S., Milosev, M. \& Novakovic, M. The effects of microwave radiation on microbial cultures. Hosp. Pharmacol. - Int. Multidiscip. J. 1, 102-108 (2014). 

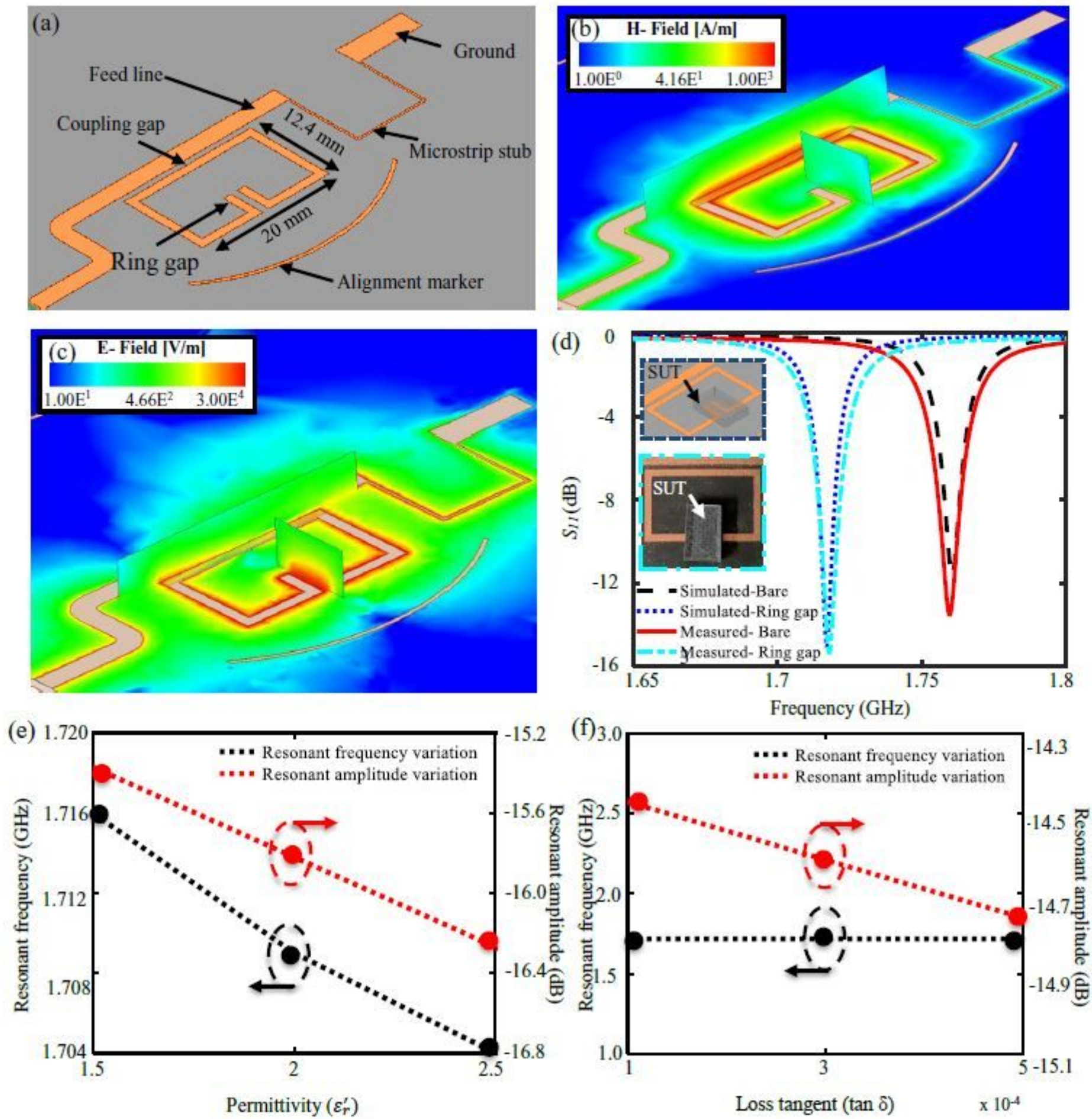

\section{Figure 1}

a) Modelled High frequency structure simulator (HFSS). b) Magnetic field distribution on the resonator. c) Electric field distribution on the resonator with the sensitive zone found in the ring gap. d) Simulated vs Measured reflection gain of the sensor in the absence and presence of a sample under test (SUT) on the 
sensitive region of the resonator. Impact of variation in e) permittivity, and f) loss tangent of the SUT on the resonant amplitude and resonant frequency of the resonator.

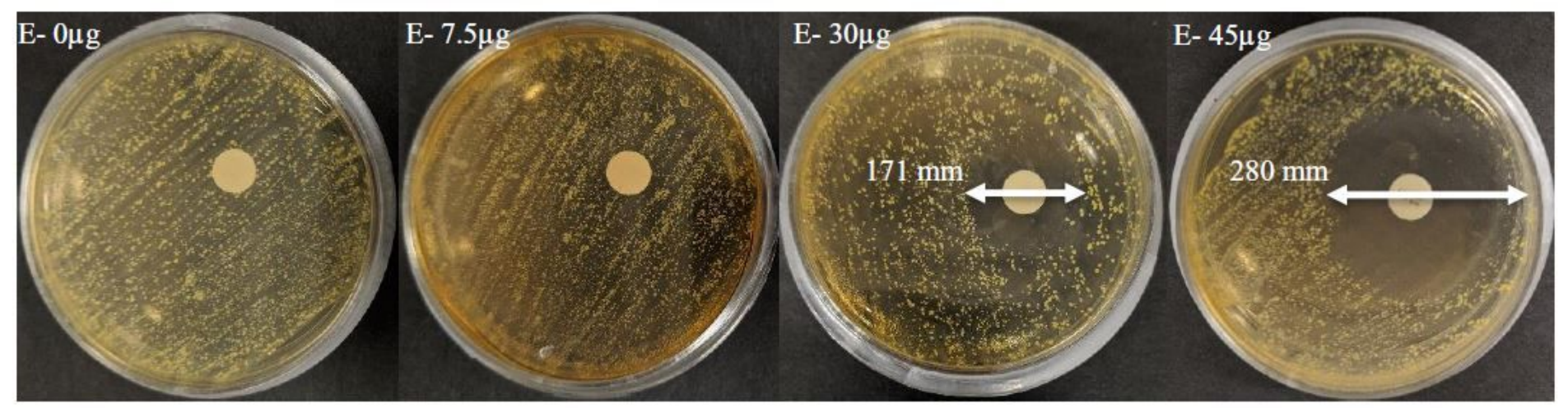

\section{Figure 2}

Captured images revealing the impact of $0,7.5,30$, and $45 \mu \mathrm{g}$ of erythromycin on the growth of E. coli. E45 , as used in this study. However, it should be noted that the bacteria is more susceptible to E- 45 than E30 .
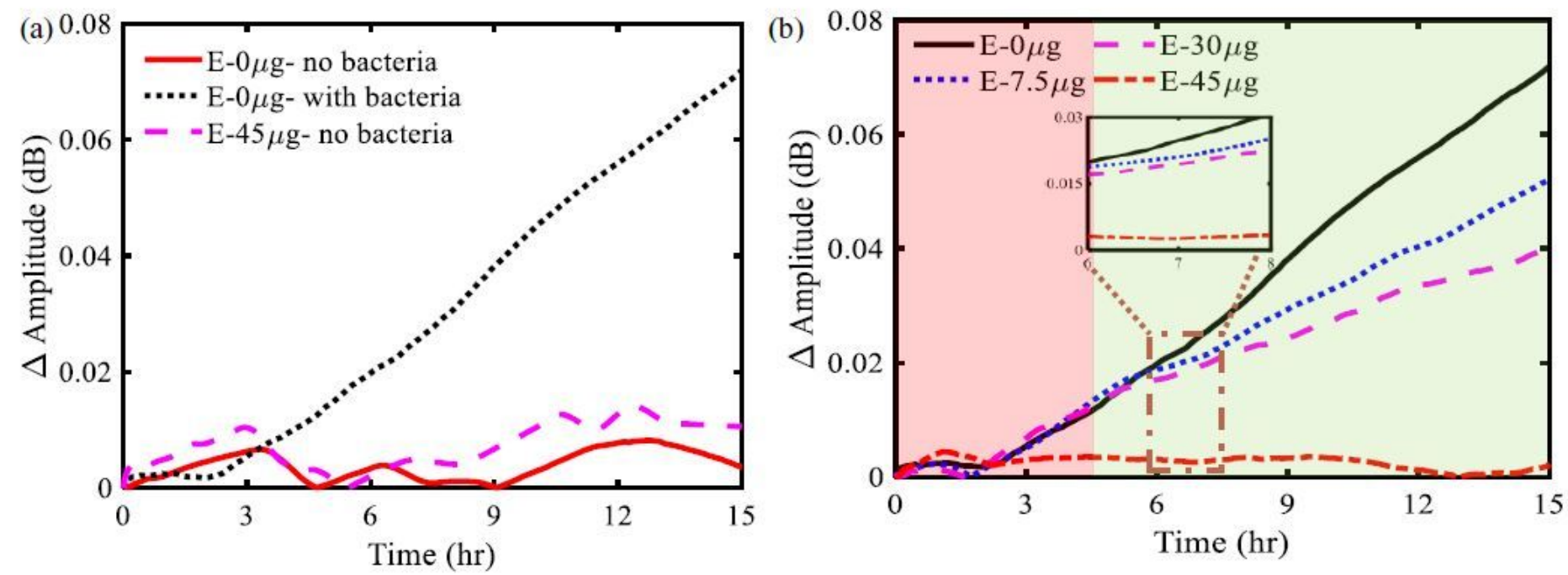

(c) $\mathrm{E}-0 \mu \mathrm{g}$

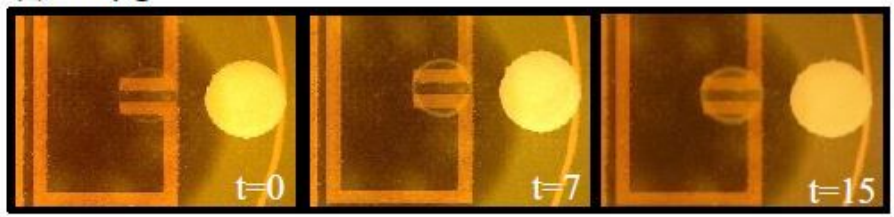

(d) E-7.5 $\mathrm{g}$

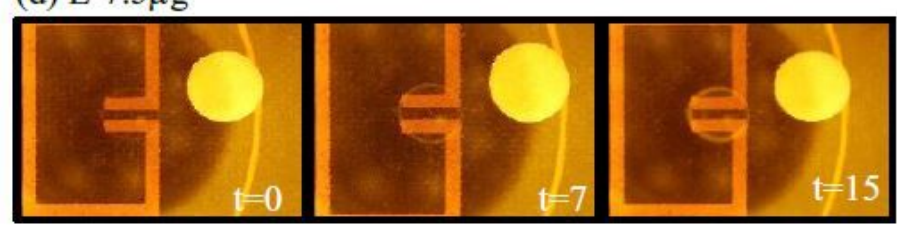

(e) E- $30 \mu \mathrm{g}$

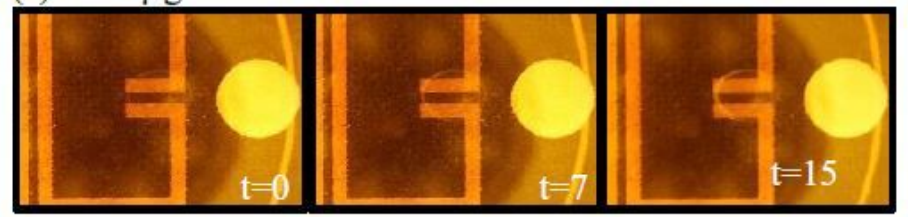

(f) $\mathrm{E}-45 \mu \mathrm{g}$

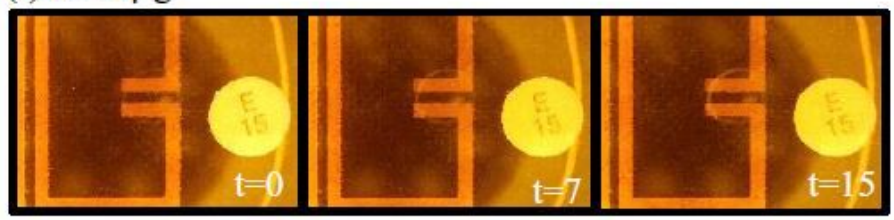

Figure 3 
a) Impact of blank paper disk (without antibiotics) and a $45 \mu$ g erythromycin disk on the response of the resonator. b) $\Delta$ resonant amplitude (dB) vs time (hr) depicting the detectable (green) and undetectable (red) regions of bacterial growth, (c)-(f) microscope images, of $0,7.5,30$, and $45 \mu \mathrm{g}$ of erythromycin.
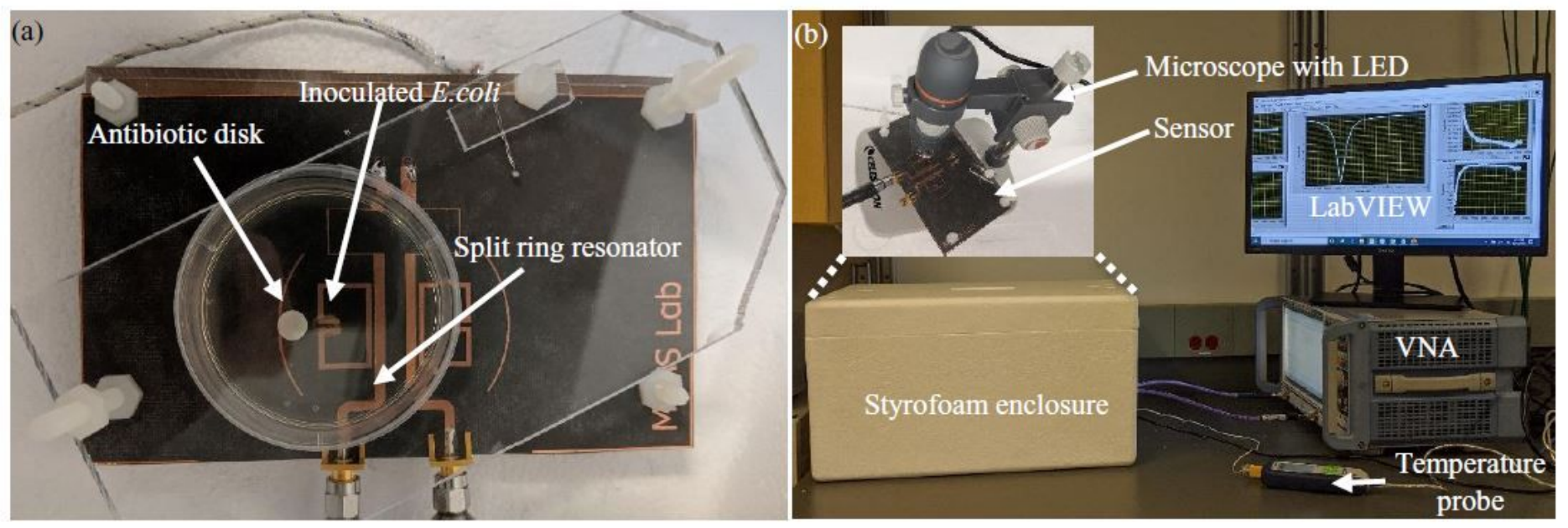

\section{Figure 4}

Fabricated sensor arrangement and the experimental setup for measurement of bacterial growth against antibiotics. a) The Fabricated planar microstrip resonator with sample petri dish placed strategically on the resonator. b) Experimental setup consisting of a thermally insulated Styrofoam enclosure equipped with sensor, temperature probe, microscope with LED, and vector network analyzer (VNA). 